\title{
Delay and Slew Metrics for On-Chip VLSI Interconnect
}

\author{
Rajib Kar, Anuran Chattaraj, Aniruddha Chandra, Ashis K. Mal, and Anup K. Bhattacharjee
}

\begin{abstract}
In deep sub-micrometer (DSM) regime the on-chip interconnect delay is significantly more dominating than the gate delay. Several approaches have been proposed to capture the interconnect delay accurately and efficiently. By interpreting the impulse response of a linear circuit as a Probability Distribution Function (PDF), Elmore first estimated the interconnect delay. Several other approaches like PRIMO, AWE, $\boldsymbol{h}$-Gamma, WED, D2M etc. have been reported so far, which are shown to be more accurate delay estimation compared to Elmore delay metric. But they suffer from computational complexity when using in the total IC design processes. On the other hand slew rate determines the ability of a device to handle the varying signals. Determination of the slew rate to a good proximity is thus essential for efficient design of high speed CMOS integrated circuits. This in turn estimates the output switching surges in the device. Interconnect slew has become a crucial bottleneck for any high density and high speed VLSI circuits as increased slew results in the increase in delay. Our work presents a closed form formulae for interconnect delay and slew calculation. The proposed metrics are derived by matching circuit moments to the Weibull distribution. This avoids use of complex look-up tables. Experiments validate the effectiveness of our metrics for nets from a real industrial design. We have achieved an average relative error as low as $15 \%$ in the delay calculation (compared to the true delay) and $4 \%$ in the slew calculation (compared to the true slew value).
\end{abstract}

Index Terms-Delay calculation, Interconnect, Moment matching, Slew calculation, Weibull Distribution function.

\section{INTRODUCTION}

As the process technology shrinks into nanometer regime, interconnect delay dominates over the gate delay; hence interconnect delay computation is becoming the crucial bottleneck for both performance and physical design optimization for high speed CMOS integrated circuits. The Elmore Delay [1] which is the first moment of the impulse response provides standard delay estimation for performance driven design applications. Elmore approximated the median of the impulse response ( $50 \%$ delay of the step response) to the mean of the impulse response by noting the similarity between non-negative impulse response and probability density functions. The Elmore delay metric has been

Manuscript received August 20, 2009.

R. Kar, A. Chattaraj, A. Chandra, A. K. Mal, and A. K. Bhattacharjee are with the Department of Electronics and Communication Engineering, National Institute of Technology, Durgapur-713209, WB, India (phone: +91-343-2755514; fax: +91-343-2547375; e-mail: rajibkarece@gmail.com, anuran_nitdgp@yahoo.co.in, toakmal@gmail.com, akbece12@yahoo.com). incredibly popular because of its simplicity, closed-form and easy to estimate. The inaccuracy of the Elmore metric arises due to the fact that it doesn't consider the resistive shielding effect of the interconnect.

In order to estimate the delay accurately and efficiently several works have been reported so far. Rubenstein et al. [2] proposed a simple closed-form formula for computing the mean of the impulse response of RC interconnect trees. Alpert et al. [3] proposed the D2M metric which is a simple function of the first two circuit moments. The PRIMO [4], $h$-Gamma [5], and WED [6] metrics are based on matching the moments of the impulse response to a particular continuous probability distribution function (PDF). PRIMO and h-Gamma match moments of the impulse response to the Gamma distribution, while WED matches to the Weibull distribution. The approaches proposed in [4-6] require some type of table lookup operation. In order to improve the accuracy of the Elmore delay metric, Asymptotic Waveform Evaluation (AWE) is being proposed [10] by matching the higher order moments of the impulse response. As the technology is shrinking towards the ultra deep sub micrometer (DSM) regime and transistor density in the chip is increasing, the length of the interconnect is getting longer. So efficient and accurate computation of the interconnect delay has become increasingly critical. On the other hand very few approaches [1] [11] have been proposed for interconnect slew calculation. In the nanotechnology age, as ultra deep sub-micron effects continue to wreak havoc on the integrity of the signal, so efficient and accurate computation of the slew metric has become critical.

We present a closed form delay and slew metrics based on the Weibull distribution. Unlike [4]-[6], matching to the Weibull distribution produces closed form formulae and no look-up table is required to compute the delay and slew.

We make the following contributions: A simple delay metric WbD (Weibull delay) and a slew metric WbS (Weibull Slew) are derived using the first two moments of the impulse response. The $\mathrm{WbD}$ and $\mathrm{WbS}$ metrics can be extended to ramp inputs using the PERI method [8]. The effectiveness of the Weibull metrics is confirmed on nets from an industrial design.

The rest of the paper is organized as follows. Section II, explains the definition and background of the circuit moments. Section III describes the properties of Weibull Distribution Section IV shows the proposed delay metric. Section V describes the proposed slew metric. Section VI presents the simulation results and comparisons with the other established 
matrices. Finally section VII concludes the paper.

\section{BASIC THEORY}

Assume that $h(t)$ is the impulse response of a node voltage in an RC circuit. The circuit moments of the impulse response are [7]:

$$
m_{k}=\frac{(-1)^{k}}{k !} \int_{0}^{\infty} t^{k} h(t) d t
$$

where $k=1,2,3 \ldots$ and $m_{k}$ is the $k^{\text {th }}$ circuit moment of the impulse response.

The circuit moments can be computed directly as functions of the RC's in time domain e.g., via path tracing algorithm. From [2] the impulse response $h(t)$ satisfies the following conditions:

$$
h(t) \geq 0 \quad \text { and } \quad \int_{0}^{\infty} h(t) d t=1
$$

Consequently, the impulse response is a probability distribution function (PDF).

The mean of the impulse response is:

$$
\mu=\int_{0}^{\infty} t h(t) d t
$$

Elmore [1] showed that $\mu=-m_{1}$ and therefore approximated the median (the desired delay) by the mean of the impulse response. We let $\mathrm{ED}=\mu=-m_{1}$, denote the Elmore delay.

The $k$ th central moment is given by:

$$
\mu_{k}=\int_{0}^{\infty}(t-\mu)^{k} h(t) d t
$$

The variance $\left(\sigma^{2}\right)$ of the impulse response can be expressed in terms of the central moments and also the circuit moments [12]:

$$
\sigma^{2}=\mu_{2}=2 m_{2}-m_{1}^{2}
$$

The key idea behind our delay and slew metrics is to match the mean and variance of the impulse response as available from(1)-(4) to those of the Weibull distribution. This calls for a detailing on the said distribution as will be pointed out in the next section.

\section{Properties Of Weibull Distribution}

The Weibull distribution is a two-parameter continuous distribution (4-43) [13]. The Weibull distribution is well suited to match the impulse response since both are unimodal and have nonnegative skewness. The Weibull PDF is given by:

$$
P(T, \eta, \beta)=\frac{\beta}{\eta}\left(\frac{T}{\eta}\right)^{\beta-1} \exp \left(-\left[\frac{T}{\beta}\right]^{\beta}\right)
$$

where $\eta>0$ and $\beta>0$ are the scale and shape parameters, respectively. Its cumulative density function $(\mathrm{CDF})$ is given by:

$$
D(T, \eta, \beta)=1-\exp \left(-\left[\frac{T}{\eta}\right]^{\beta}\right)
$$

The expected value (or mean) and the variances are, respectively, given by:

$$
E(x)=\eta \Gamma\left(\frac{1}{\beta}+1\right)
$$

and,

$$
\operatorname{Var}(x)=\eta\left\{\Gamma\left(\frac{2}{\beta}+1\right)-\Gamma^{2}\left(\frac{1}{\beta}+1\right)\right\}
$$

\section{Proposed Delay Metric}

One can match two common properties of the Weibull distribution and the circuit's impulse response. Note that the mean and variance of the impulse response are $\mu=-m_{1}$ and $\sigma^{2}=2 m_{2}-m_{1}^{2}$, respectively. Using (8) and (9), by matching the mean and variance yields,

$$
\eta \Gamma\left(\frac{1}{\beta}+1\right)=-m_{1}
$$

and

$$
\eta\left\{\Gamma\left(\frac{2}{\beta}+1\right)-\Gamma^{2}\left(\frac{1}{\beta}+1\right)\right\}=2 m_{2}-m_{1}^{2}
$$

Let us consider a variable $\alpha$ such that $\alpha=1 / \beta$. We approximate the Gamma function as (6.1.39) [14],

$$
\Gamma(a z+b) \approx \sqrt{2 \pi} \exp (-a z)(a z)^{a z+b-\frac{1}{2}}
$$

Now solving (10) and (11) using (12) yields,

$$
\alpha=\left(\frac{1}{\ln 16}\right) \ln \left(\frac{4 \pi m_{2}^{2}}{m_{1}^{4} \ln 16}\right)
$$

and,

$$
\eta=\frac{m_{1}}{\Gamma(\alpha+1)}=\frac{m_{1}}{\sqrt{2 \pi} \exp (-\alpha)(\alpha)^{\alpha+1 / 2}}
$$

Quite naturally from (13) we can compute $\beta=1 / \alpha$ as,

$$
\beta=\frac{\ln 16}{\ln \left(\frac{4 \pi m_{2}^{2}}{m_{1}^{4} \ln 16}\right)}
$$

Note that the median of the Weibull Distribution is given by

$$
M=\eta[\ln (2)]^{1 / \beta}
$$

One can verify this by setting $D(T, \eta, \beta)=0.5$ in (7) and solving for $T$. Thus, when matching the impulse response the median becomes our $50 \%$ delay metric. Substituting $\eta$ and $\beta$ values from (14) and (15) in (16) we finally get the delay metric as:

$$
W b D=m_{1} \frac{[\ln (2)]^{\alpha}}{\sqrt{2 \pi} \exp (-\alpha) \alpha^{\alpha+1 / 2}}
$$

The above proposed delay function is in closed-form and simple enough in that it involves first two circuit moments only. 


\section{Proposed Slew Metric}

Let $T_{\mathrm{LO}}$ and $T_{\mathrm{HI}}$ be $10 \%$ and $90 \%$ delay points respectively. Matching these points to the CDF yields:

$$
0.1=1-\exp \left(-\left[\frac{T_{\mathrm{LO}}}{\eta}\right]^{\beta}\right)
$$

and,

$$
0.9=1-\exp \left(-\left[\frac{T_{\mathrm{HI}}}{\eta}\right]^{\beta}\right)
$$

From (18) and (19), we have

$$
T_{\mathrm{LO}}=\eta\left[\ln \left(\frac{10}{9}\right)\right]^{1 / \beta}
$$

and,

$$
T_{\mathrm{HI}}=\eta[\ln (10)]^{1 / \beta}
$$

Finally using (20) and (21) the Weibull slew metric can be written as,

$$
W b S=\frac{T_{\mathrm{HI}}-T_{\mathrm{LO}}}{t_{\mathrm{HI}}-t_{\mathrm{LO}}}=m_{1} \frac{[\ln (10)]^{\alpha}-\left[\ln \left(\frac{10}{9}\right)\right]^{\alpha}}{\sqrt{2 \pi} t \exp (-\alpha) \alpha^{\alpha+1 / 2}}
$$

\section{EXPERIMENTAL RESULTS}

In order to verify the efficiency of our model, we have extracted 200 routed nets containing 1024 sinks from an industrial ASIC design in $0.13 \mu \mathrm{m}$ technology. We choose the nets so that the maximum sink delay is at least 20 ps and the delay ratio between closet and furthest sink in the net is less than 0.2. It ensures that each net has at least one near end sink. We classify the 1024 sinks into the following three categories:

1) 511 far-end sinks have delay greater or equal to $75 \%$ of the maximum delay to the furthest sink in the net.

2) 342 mid-end sinks which have delay between $25 \%$ and $75 \%$ of the maximum delay and

3) 171 near-end sinks which have delay less than or equal to $25 \%$ of the maximum delay.

For each RC network source we put a driver, where the driver is a voltage source followed by a resister. For each sink we compute the delay and slew using SPICE simulator and measure the relative error of the appropriate metric to the SPICE result. The simulation goal is twofold: first, to compute our delay metric (WbD) with Kahng-Muddu Model (KM) [9] and Elmore Delay (ED [1] and second, to assess our slew metric (WbS) in comparison to [1] and [11]. We call these metrics as $\mathrm{ES}$ and $\mathrm{BkS}$, respectively.

Table 1 lists the results with our proposed delay metric (WbD) along with KM and ED. It shows that our approach leads to an average error of less than $15 \%$ compared to the true delay value for lower value of driver resistance. From Table 2, we find that our proposed model provides the best slew estimation compared to other approaches and results an average error of less than $4 \%$ for higher value of driver resistance.

\section{CONCLUSIONS}

We have proposed $\mathrm{WbD}$, a closed form delay metric and $\mathrm{WbS}$, closed form slew metric for RC trees that is a simple function of two moments of the impulse response, for performance optimization. Our metric has the Elmore delay as a theoretical upper bound, but with significantly less error. $\mathrm{WbD}$ is more accurate than $\mathrm{KM}$ and is indeed remarkably accurate at the near end. $\mathrm{WbD}$ has the advantage that its Elmore-like formula may make it more suitable and accurate for optimization purposes. $\mathrm{WbS}$ is more accurate than BakS and accurate at the far end nodes.

\section{REFERENCES}

[1] W. C. Elmore, "The Transient Response of Damped Linear Network with Particular Regard to Wideband Amplifiers,” J. Applied Physics, vol. 19, 1948, pp. 55-63.

[2] J. Rubenstein, P. Penfield, and M. A. Horowitz, "Signal Delay in RC Tree Networks," IEE Trans. CAD-2, July 1983.

[3] C. J. Alpert, A. Devgan, and C. Kashyap, "RC Delay Metrics for Performance Optimization,” IEEE Trans. CAD, vol. 20, no. 5, 2001, pp. 571-582.

[4] R. Kay and L. Pileggi, "PRIMO: Probability Interpretation of Moments for Delay Calculation," IEE WACM Design Automation Conference, 1998, pp. 463-468.

[5] T. Lin, E. Acar, and L. Pileggi, "h-gamma: An RC Delay Metric Based on a Gamma Distribution Approximation to the homogeneous Response," IEEE/ACM International Conference on Computer-Aided Design, 1998, pp. $19-25$.

[6] F. Liu, C. Kashyap, and C. J. Alpert, “A Delay Metric for RC Circuits based on the Weibull Distribution", IEEE/ACM Intl. Conference on Computer-Aided Design, 2002, pp. 620-624.

[7] M. Celik, L. Pileggi, A. Odabasioglu, IC Interconnect Analysis. Kluwer Academic Publishers, 2002

[8] C. Kashyap, C. J. Alpert, E Liu, and A. Devgan, "PERI: A Technique for Extending Delay and Slew Metrics to Ramp Input," ACM Symposium on Physical Design, 2003.

[9] A. B. Kahng and S. Muddu, "An Analytical Delay Model for RLC Interconnects,” IEEE Trans. CAD, vol. 16, no. 12, 1997, pp. 1507-1514.

[10] L. T. Pillage and R. A. Rohrer, "Asymptotic Waveform Evaluation for Timing Analysis,” IEEE Trans. CAD, vol. 9, no. 4, 1990, pp. 352-366.

[11] H. B. Bakoglu, Circuits, Interconnects, and Packaging for VLSI. Addison-Wesley Publishing Company, 1990.

[12] R. Gupta, B. Tutuianu, and L. T. Pileggi, "The Elmore Delay as a Bound for RC Trees with Generalized Input Signals," IEEE Trans. CAD, vol. 16, no. 1,1997 , pp. 95-104.

[13] A. Papoulis and S. U. Pillai, Probability, Random Variables and Stochastic Processes. 4th ed., New Delhi: Tata McGraw Hill, 2006.

[14] M. Abramowitz and I. A. Stegun, Handbook of Mathematical Functions with Formulas, Graphs and Mathematical Tables. 9th ed., New York: Dover, 1970. 
Table 1. Comparison for Weibull Delay Metric

\begin{tabular}{|c|c|c|c|c|c|c|}
\hline \multicolumn{7}{|c|}{ Driver resistance $=0 \Omega$} \\
\hline & \multicolumn{3}{|c|}{ Average \% Relative error } & \multicolumn{3}{|c|}{ \% Standard Deviation } \\
\hline Sink & WbD & ED & KM & WbD & ED & KM \\
\hline Near & 45.36 & 268.72 & 113.87 & 33.78 & 166.37 & 82.9 \\
\hline Mid & 14.72 & 69.86 & 15.97 & 10.38 & 27.27 & 12.27 \\
\hline Far & 1.03 & 26.912 & 1.048 & 0.952 & 2.864 & 1.32 \\
\hline Total & 14.51 & 90.96 & 29.744 & 24.83 & 118.88 & 59.81 \\
\hline \multicolumn{7}{|c|}{ Driver resistance $=100 \Omega$} \\
\hline & \multicolumn{3}{|c|}{ Average \% Relative error } & \multicolumn{3}{|c|}{ \% Standard Deviation } \\
\hline Sink & WbD & ED & KM & WbD & ED & KM \\
\hline Near & 87.6 & 416.78 & 197.36 & 54.31 & 246.29 & 131.81 \\
\hline Mid & 14.56 & 64.98 & 15.11 & 9.07 & 22.42 & 11.58 \\
\hline Far & 01.192 & 29.248 & 1.32 & 0.904 & 3.302 & 1.032 \\
\hline Total & 21.41 & 114.56 & 42.08 & 39.74 & 183.81 & 94.3 \\
\hline \multicolumn{7}{|c|}{ Driver resistance $=\mathbf{2 0 0 \Omega}$} \\
\hline & \multicolumn{3}{|c|}{ Average \% Relative error } & \multicolumn{3}{|c|}{ \% Standard Deviation } \\
\hline Sink & WbD & ED & KM & WbD & ED & KM \\
\hline Near & 99.01 & 366.32 & 182.72 & 50.39 & 182.77 & 177.7 \\
\hline Mid & 11.912 & 59.62 & 12.184 & 6.776 & 18.496 & 11.288 \\
\hline Far & 1.32 & 27.736 & 1.352 & 0.696 & 2.936 & 1.176 \\
\hline Total & 22.14 & 101.28 & 36.34 & 31.87 & 151.28 & 36.152 \\
\hline
\end{tabular}

Table 2. Comparison for Weibull Slew Metric

\begin{tabular}{|c|c|c|c|c|c|c|}
\hline \multicolumn{7}{|c|}{ Driver resistance $=0 \Omega$} \\
\hline & \multicolumn{3}{|c|}{ Average \% Relative error } & \multicolumn{3}{|c|}{ \% Standard Deviation } \\
\hline Sink & BakS & ES & WbS & BakS & ES & WbS \\
\hline Near & 65.45 & 786.13 & 43.72 & 44.25 & 615.10 & 27.38 \\
\hline Mid & 11.76 & 24.27 & 4.65 & 7.832 & 23.62 & 4.59 \\
\hline Far & 9.23 & 11.23 & 2.831 & 6.96 & 10.30 & 3.1 \\
\hline Total & 21.46 & 144.12 & 14.67 & 34.67 & 401.1 & 21.85 \\
\hline \multicolumn{7}{|c|}{ Driver resistance $=100 \Omega$} \\
\hline & \multicolumn{3}{|c|}{ Average \% Relative error } & \multicolumn{3}{|c|}{ \% Standard Deviation } \\
\hline Sink & BakS & ES & WbS & BakS & ES & WbS \\
\hline Near & 17.25 & 143.23 & 15.34 & 18.3 & 98.9 & 15.66 \\
\hline Mid & 12.3 & 31.2 & 7.87 & 10.23 & 26.56 & 7.67 \\
\hline Far & 9.45 & 16.6 & 6.78 & 7.12 & 14.43 & 4.56 \\
\hline Total & 10.35 & 29.4 & 7.23 & 10.54 & 78.65 & 6.98 \\
\hline \multicolumn{7}{|c|}{ Driver resistance $=200 \Omega$} \\
\hline & \multicolumn{3}{|c|}{ Average \% Relative error } & \multicolumn{3}{|c|}{ \% Standard Deviation } \\
\hline Sink & BakS & ES & WbS & BakS & ES & WbS \\
\hline Near & 11.43 & 84.39 & 6.5 & 21.31 & 105.12 & 17.82 \\
\hline Mid & 13.1 & 34.68 & 4.53 & 7.22 & 21.29 & 3.14 \\
\hline Far & 8.08 & 9.76 & 1.93 & 6.96 & 13.14 & 1.9 \\
\hline Total & 9.135 & 16.269 & 3.41 & 10.97 & 22.11 & 5.11 \\
\hline
\end{tabular}

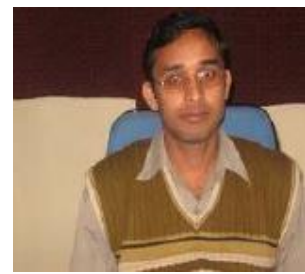

Rajib Kar received his B. E. degree in electronics and communication engineering and M. Tech. degree in communication engineering from NIT (formerly R. E. College), Durgapur, India in 2001 and 2008, respectively and is currently pursuing a $\mathrm{Ph}$. D. there.

$\mathrm{He}$ joined electronics and communication engineering department, National Institute of Technology (NIT), Durgapur in 2002 as a Lecturer. His research interests include interconnect analysis and synthesis, mixed signal IC analysis, and low power VLSI.

Mr. Kar has published 18 research papers in reputed journals and peer-reviewed conferences. He is a member of IAENG and has also served as a reviewer for a number of peer reviewed journals and conferen
Anuran Chattaraj received his B. E. degree in electronics and communication engineering from NIT Durgapur, India in 2009.

His research interests include RFID and interconnect analysis.

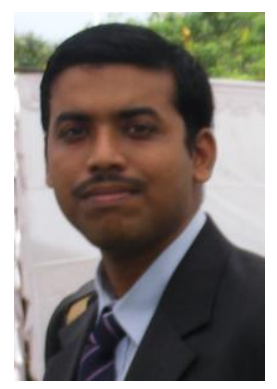

Aniruddha Chandra received his B. E. (Hons.) degree in electronics and communication engineering and M. E. degree in communication engineering from Jadavpur University (JU), Kolkata, India in 2003 and 2005 respectively and is currently pursuing a $\mathrm{Ph}$. D. there.

$\mathrm{He}$ joined electronics and communication engineering department, National Institute of Technology (NIT) Durgapur in 2005 as a Lecturer. His research interests include diversity combining, modulation techniques, and application of statistics.

Mr. Chandra has co-authored a book titled Analog Electronic Circuits and published 20 research papers in reputed journals and peer-reviewed conferences. He has also delivered several invited lectures including IEEE comsoc lecture meetings at JU, Kolkata. He received the prestigious NTSE and EFIP scholarships from NCERT and MHRD, Govt. of India respectively. He is a member of IEEE (Communication Society), IAENG and has also served as a reviewer for IEEE Potentials and Computer and Electrical Engineering (Elsevier). 


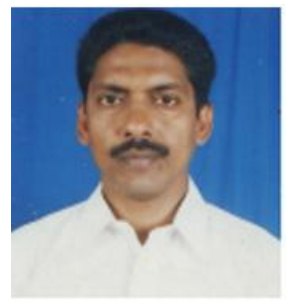

Ashis Kumar Mal received his B. E., M. Tech and Ph. D. degrees in 1989, 1994 and 2009, respectively.

$\mathrm{He}$ joined electronics and communication engineering department, National Institute of Technology (NIT) Durgapur in 2007 as an Assistant Professor. His research interests include interconnect analysis and synthesis, mixed signal IC analysis, and low power VLSI.

Dr. Mal has published 25 research papers in reputed journals and peer-reviewed conferences. He is a member of IEEE and has also served as a reviewer for a number of peer reviewed journals and conferences.

Anup Kumar Bhattacharjee received his B. E., M. E. and Ph. D. degrees in 1983, 1985 and 1989, respectively.

He joined electronics and communication engineering department, National Institute of Technology (NIT) (formerly R.E.College) Durgapur in 1988 as Lecturer. Dr. Bhattacharjee is currently serving in the capacity of Professor at ECE Department, NIT Durgapur. His research interests include micro strip antenna, microwave engineering, and VLSI systems.

Dr. Bhattacharjee has published 45 research papers in reputed journals and peer-reviewed conferences. He has also served as a reviewer for a number of peer reviewed journals and conferences. 